Dirkhäuser 

Carlos S. Kubrusly

\section{Spectral Theory of Operators on Hilbert Spaces}


Carlos S. Kubrusly

Electrical Engineering Department

Catholic University of Rio de Janeiro

Rio de Janeiro, RJ, Brazil

ISBN 978-0-8176-8327-6

e-ISBN 978-0-8176-8328-3

DOI 10.1007/978-0-8176-8328-3

Springer New York Dordrecht Heidelberg London

Library of Congress Control Number: 2012939729

Mathematics Subject Classification (2010): 47-XX. 47Axx

(C) Springer Science+Business Media, LLC 2012

All rights reserved. This work may not be translated or copied in whole or in part without the written permission of the publisher (Springer Science+Business Media, LLC, 233 Spring Street, New York, NY 10013, USA), except for brief excerpts in connection with reviews or scholarly analysis. Use in connection with any form of information storage and retrieval, electronic adaptation, computer software, or by similar or dissimilar methodology now known or hereafter developed is forbidden.

The use in this publication of trade names, trademarks, service marks, and similar terms, even if they are not identified as such, is not to be taken as an expression of opinion as to whether or not they are subject to proprietary rights.

Printed on acid-free paper

Springer is part of Springer Science+Business Media

(www.birkhauser-science.com) 
To $A \& J \& A \& A \& B$ 
... and all life long my instinct has been to abandon anything for which I have no talent; tennis, golf, dancing, sallying, all have been abandoned, and perhaps it is desperation which keeps me writing...

Graham Greene 


\section{Preface}

This work is an introduction to the spectral theory of Hilbert space operators. My main goal is to offer a modern introductory textbook for a first course in spectral theory for graduate students, emphasizing recent aspects of the theory, with detailed proofs. The book is addressed to a wide audience consisting of graduate students in mathematics, as well as those studying statistics, economics, engineering, and physics. The text, however, can also be useful to working mathematicians delving into the spectral theory of Hilbert space operators, and for scientists wishing to apply spectral theory to their field.

The prerequisite for this book is a first course in elementary functional analysis including, in particular, operator theory on Hilbert spaces at an introductory level. Of course, this includes a formal introduction to analysis, covering elements of measure theory and functions of a complex variable. However, every effort has been made to ensure that this book, despite its relative shortness, is as self-contained as possible.

Chapter 1 summarizes the basic concepts from Hilbert space theory that will be required in the text. By no means is it intended to replace a formal introductory course on single operator theory. Its main purpose, besides summarizing the basics required for further chapters, is to unify notation and terminology. Chapter 2 discusses standard spectral results for (bounded linear) operators on Banach and Hilbert spaces, including the classical partition of the spectrum and spectral properties for specific classes of operators. Chapter 3 is devoted wholly to the Spectral Theorem for normal operators. After considering the compact case, it meticulously treats the general case, carrying out the proofs of both versions of the Spectral Theorem for the general case in great detail. The idea behind those highly detailed proofs is to discuss and explain delicate points, stressing some usually hidden features. The chapter closes with the Fuglede Theorems. Chapter 4 deals with functional calculus for normal operators, which depends on the Spectral Theorem, and also with analytic functional calculus (i.e., Riesz functional calculus). The same level of detail employed in the proofs of Chapter 3 is repeated here. Chapter 5 focuses 
on Fredholm theory and compact perturbations of the spectrum, where a finer analysis of the spectrum is worked out, leading to further partitions involving the essential spectrum, the Weyl spectrum, and the Browder spectrum. The chapter closes with a discussion of Weyl's and Browder's Theorems, including some very recent results.

The final section of each chapter is a section on Additional Propositions, consisting of either auxiliary results that will be required to support a proof in the main text, or further results extending some theorems proved in the main text. These are followed by a set of Notes, where each proposition is briefly discussed, and references are provided indicating proofs for all of them. The Additional Propositions can also be thought of as a set of proposed problems, and their respective Notes can be viewed as hints for solving them.

At the end of each chapter, the reader will find a collection of suggested readings. This has a triple purpose: to offer a reasonable bibliography including most of the classics as well as some quite recent texts; to indicate where different approaches and proofs can be found; and to indicate where further results can be found. In this sense, some of the references are suggested as a second reading on the subject.

The material in this book has been prepared to be covered in a onesemester graduate course. The resulting text is the outcome of attempts to meet the needs of a contemporary first course in spectral theory for an audience as described in the first paragraph, possessing the prerequisites listed in the second paragraph of this Preface. The logical dependence of the various sections (and chapters) is roughly linear and reflects approximately the minimum amount of material needed to proceed further.

I have been lecturing on this subject for a long time; I often present it as a second part of an operator theory course. As a result, I have benefited from the help of many friends, among students and colleagues, and I am very grateful to all of them. In particular, I wish to thank João Zanni for his painstaking quest for typos. I also wish to thank Torrey Adams who copyedited the manuscript. Special thanks are due to an anonymous referee who made significant contributions throughout the text, and who corrected some important inaccuracies and mistakes that existed in the original version. Thanks are also due to Catholic University of Rio de Janeiro for providing the release time that made this project possible, as well as to CNPq (Brazilian National Research Council) for a research grant.

Rio de Janeiro, March 2012

Carlos S. Kubrusly 


\section{Contents}

Preface

1 Preliminaries $\quad 1$

1.1 Notation and Terminology $\ldots \ldots \ldots \ldots \ldots \ldots \ldots \ldots \ldots \ldots \ldots$

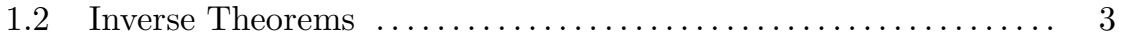

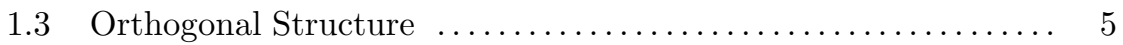

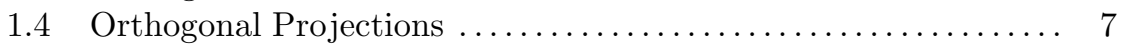

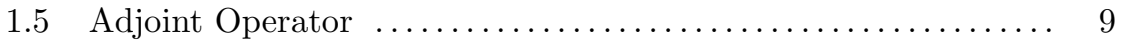

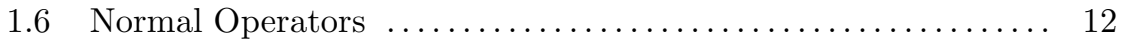

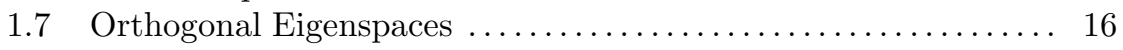

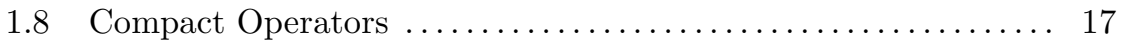

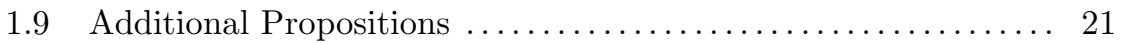

2 Spectrum $\quad 27$

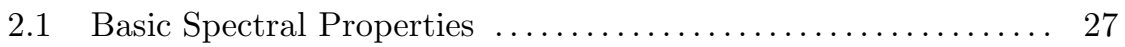

2.2 A Classical Partition of the Spectrum ................ 30

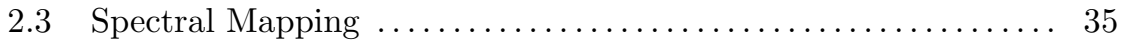

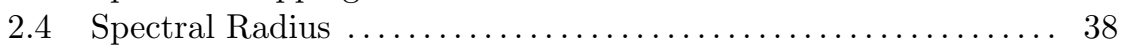

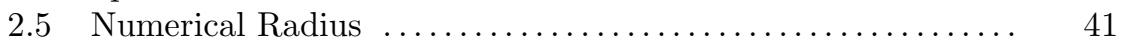

2.6 Spectrum of Compact Operators ........................ 44

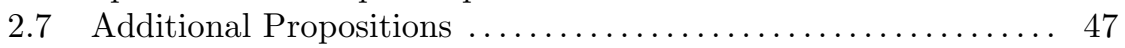

3 Spectral Theorem $\quad 55$

3.1 Spectral Theorem for Compact Operators .............. 55

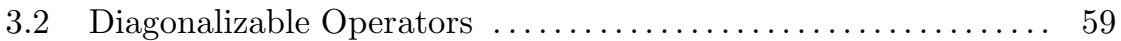

3.3 Spectral Measure ........................... 63

3.4 Spectral Theorem: General Case .................... 67

3.5 Fuglede Theorems and Reducing Subspaces ............. 79

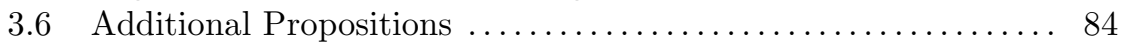


4 Functional Calculus $\quad 91$

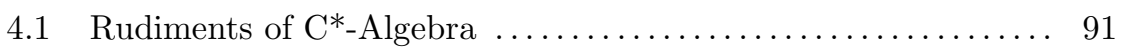

4.2 Functional Calculus for Normal Operators ............... 95

4.3 Analytic Functional Calculus: Riesz Functional Calculus ..... 102

4.4 Riesz Decomposition Theorem and Riesz Idempotents ...... 115

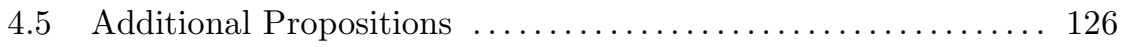

5 Fredholm Theory 131

5.1 Fredholm Operators and Fredholm Index .............. 131

5.2 Essential Spectrum and Spectral Picture ................ 141

5.3 Riesz Points and Weyl Spectrum .................... 153

5.4 Ascent, Descent, and Browder Spectrum .................. 162

5.5 Remarks on Browder and Weyl Theorems .............. 178

5.6 Additional Propositions ....................... 184

$\begin{array}{ll}\text { References } & 187\end{array}$

$\begin{array}{ll}\text { Index } & 193\end{array}$ 